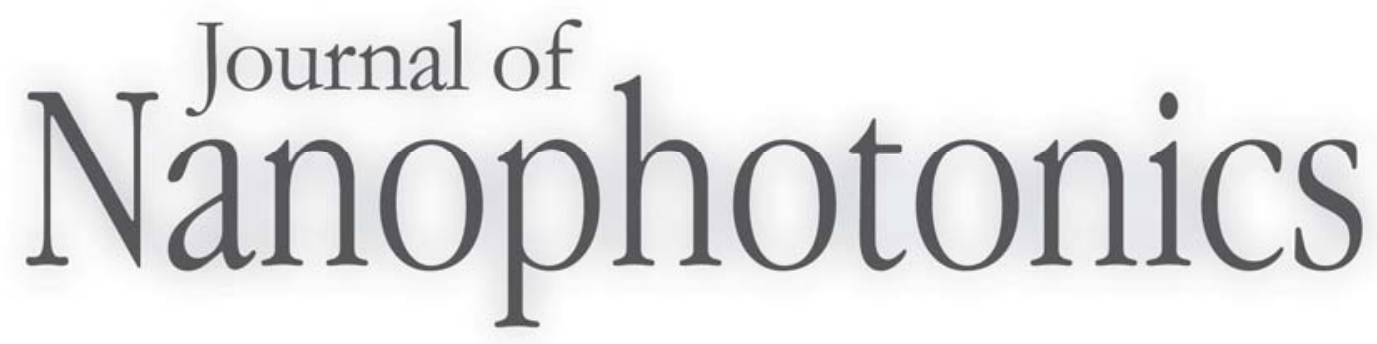

SPIEDigitalLibrary.org/jnp

\title{
Commentary: \\ New developments in single photon detection in the short wavelength infrared regime
}

Robert J. Collins

Robert H. Hadfield

Gerald S. Buller 


\title{
New developments in single photon detection in the short wavelength infrared regime
}

\author{
Robert J. Collins, Robert H. Hadfield, and Gerald S. Buller \\ Heriot-Watt University, School of Engineering and Physical Sciences, \\ David Brewster Building, Riccarton, Edinburgh, UK, EH14 4AS \\ r.j.collins@hw.ac.uk,r.h.hadfield@hw.ac.uk, g.s.buller@hw.ac.uk
}

Many new and exciting fields of scientific research rely on the efficient detection of single photons in the infrared regime. To give just two examples, quantum key distribution has the potential to offer verifiably secure methods of generating cryptographic keys and active imaging techniques permit the creation of detailed 3D models of distant objects. Currently, many of these technologies use photons in the wavelength range 400 to $1000 \mathrm{~nm}$ due to the ready availability of relatively efficient single photon detectors for use at these wavelengths (recent surveys may be found in Refs. [1] and [2]). However, extending the wavelength range to $1.3 \mu \mathrm{m}$ and $1.55 \mu \mathrm{m}$ would result in many benefits, for example compatibility with lowloss optical fiber spectral regions and atmospheric transmission windows, as well as infrared gas absorption features, notably those of greenhouse gases. Let us briefly consider the current status of the potential single photon detection technologies available for this spectral region.

Currently, there are three main types of detector technology in widespread use; photomultipliers, semiconductor avalanche photodiodes and superconducting detectors. Within these different technologies, there are different material systems and micro-structure designs leading to a wide spread in key performance characteristics for similar devices. One figure of merit for such detectors is the noise equivalent power (NEP), defined as the signal power required to attain a unity signal-to-noise ratio within a one second integration time.

Photomultiplier tubes (PMTs) operate by the cascade generation of a stream of electrons from an initial photo-generated electron. A photon incident on a photocathode will, with a probability less than unity, trigger the emission of an electron. This electron is accelerated towards an anode and, on collision, triggers the release of a number of secondary electrons. This process is repeated through a series of anodes, multiplying the total number of electrons each time, until a detectable current pulse is generated. These devices have large photocathode areas (typically of the order of a few $\mathrm{mm}^{2}$ ) and gain which depends on dynode geometry and bias. PMTs typically operate with photons at lower wavelengths than those considered in this commentary but devices with an InGaAs photocathode such as the those produced by Hamamatsu have demonstrated an NEP $\sim 4 \times 10^{-16} \mathrm{~W} \mathrm{~Hz}^{-1 / 2}$ at a wavelength of $1.55 \mu \mathrm{m}$ when cooled to $210 \mathrm{~K}$.

The most common semiconductor-based devices are single photon avalanche diode (SPAD) detectors, which are reverse biased avalanche photodiodes (APDs) biased above the avalanche breakdown voltage in the Geiger mode. In this mode of operation, a single incident photon can generate an electron-hole pair which can initiate a self-sustaining avalanche, rapidly generating a readily detectable current pulse. After each event, the avalanche current must be quenched to restore the detector to the quiescent state ready to detect further photons.

At present, detectors based on Indium-Gallium-Arsenide/Indium-Phosphide (InGaAs/InP) offer the best prospect for near room-temperature operation SPADs. Historically, devices based on this material system have been used as linear multiplication devices but more recent advances in Geiger mode operation have led to the development of a growth and fabrication process specifically targeted with the aim of producing single photon detectors [3]. 


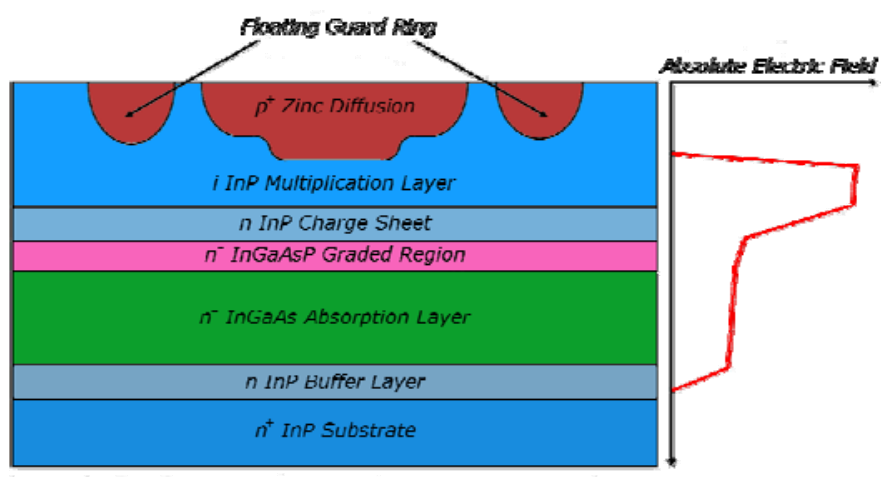

Fig. 1. An illustration of the layer structure of planar geometry InGaAs/InP single photon avalanche diode. The junction is formed by the diffusion of zinc ( $\mathrm{Zn})$ into ntype InP and typically is fabricated with multiple diffusion steps and guard rings in order to avoid preferential edge breakdown. Typical device diameters are $10-200 \mu \mathrm{m}$.

A schematic of the cross-section of a planar geometry InGaAs/InP single photon detector is illustrated in Fig. 1. Incident photons in the infrared regime are absorbed in the narrowbandgap InGaAs layer and the photo-generated holes then drift to the high-field InP layer where multiplication takes place. The thin (typically $100 \mathrm{~nm}$ ) $\mathrm{n}^{-}$InGaAsP layer serves to provide a step in the large valence band discontinuity between the InGaAs and InP layers and thereby assist the drifting holes in traversing into the multiplication region.

A significant issue with these detectors is the deleterious effects of afterpulsing, which are caused by the avalanche current filling mid-gap trap states in the material. These trap states then emit carriers at a later time, contributing further to the dark events. The resulting high afterpulsing rates can be reduced by having a bias below breakdown after each event, in order that the traps can be emptied without resulting in an avalanche pulse. The afterpulsing rate depends on temperature, gate duration and the constituent material properties and will set an upper limit on the gating rate, consequently setting limitations on the maximum count rate possible with the detector. Early experiments on InGaAs/InP single photon detectors typically used a combination of cooled operating conditions to reduce the probability of carriers traversing the band-gap and causing dark events, and a simple electrical gating technique to allow traps to empty and reduce the effects of afterpulsing.

Almost a decade ago, NEPs as low as $4 \times 10^{-17} \mathrm{~W} \mathrm{~Hz}^{-1 / 2}$ at a wavelength of $1.55 \mu \mathrm{m}$ were reported for actively quenched commercially-available linear multiplication InGaAs/InP devices operated in Geiger mode at a temperature of $77 \mathrm{~K}$, and sub-nanosecond timing jitter measurements were reported by a number of different groups at various temperatures. Many subsequent successful experiments have relied on improved quenching methods to reduce the charge flow per avalanche event in order to diminish the effects of afterpulsing. In recent years operation at a temperature of $223 \mathrm{~K}$ has been demonstrated with gating rates near, or in excess of, $1 \mathrm{GHz}$. For example, Yuan et al. [4] used a self-differencing circuit to reduce the charge flow per photon event resulting in reduced afterpulsing and operation at gigahertz clock rates. These gigahertz gating approaches can work well at near room temperature operation for applications such as quantum key distribution where the photon events occur in predefined time windows. However, applications such as photon-counting time-of-flight ranging where the photon arrival times can be less easily predetermined require an un-gated (free-running) detection technique for more efficient photon event acquisition. Free-running operation at a temperature of $210 \mathrm{~K}$ using rapid active quenching was demonstrated by Thew et al. in 2007 [5]. A greater understanding of the field-assisted tunneling in the InP, which is 
the principal dark count mechanism, has led to the introduction of longer InP multiplication regions and resulting improvements in sensitivity. Use of these devices in the low-bias regime have led to room temperature operation with no electrical gating resulting in an NEP of $\sim 1 \times 10^{-15} \mathrm{~W} \mathrm{~Hz}^{-1 / 2}$, reducing to $5 \times 10^{-17} \mathrm{~W} \mathrm{~Hz}^{-1 / 2}$ at $210 \mathrm{~K}$, with a count rate of $10^{6}$ counts per second demonstrated [6].

The third detector technology of note is a superconducting single photon detector technology (SSPD) based on a niobium nitride $(\mathrm{NbN})$ nanowires. The nanowire is cooled well below its superconducting transition temperature (to the temperature range 1.5-4 K) and biased at close to the superconducting critical current. An incident photon will generate a resistive hot-spot, perturbing the supercurrent distribution and triggering a measurable voltage pulse. This process is summarized in Fig. 2 [7]. Individual NbN nanowire SSPDs are typically based on a meander configuration covering a $100 \mu \mathrm{m}^{2}$ area, suitable for coupling with telecommunications single mode optical fiber. The superconducting energy gap of $\mathrm{NbN}$ is of the order of meV (three orders of magnitude smaller than the bandgap of a typical semiconductor). Consequently, the spectral response of the detectors extends from visible wavelengths into the mid infrared, approximately $400 \mathrm{~nm}$ to $3 \mu \mathrm{m}$. The detection efficiency and dark count rate are dependent on the bias current. Dark counts increase exponentially close to the critical current, so lowest NEP is obtained at lower bias. NbN nanowire SSPDs have been implemented in practical closed cycle refrigerator systems and used in many photon-counting applications. A practical NEP of $\sim 8 \times 10^{-17} \mathrm{~W} \mathrm{~Hz}^{-1 / 2}$ at a wavelength of 1.55 $\mu \mathrm{m}$ (detection efficiency $0.7 \%$, dark count rate $10 \mathrm{~Hz}$ ) has been demonstrated [8]. They exhibit an almost Gaussian temporal profile and typical timing jitters of less than $70 \mathrm{ps}$.

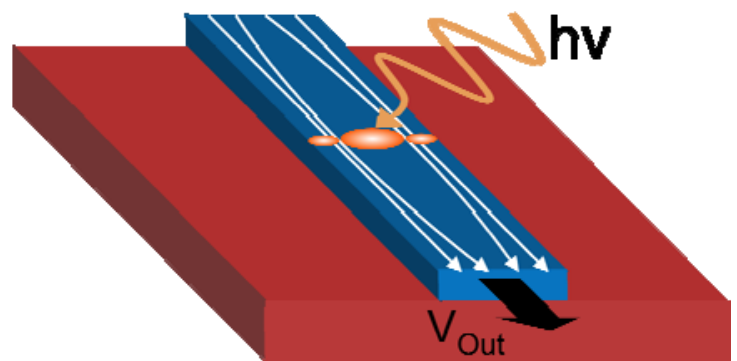

Fig. 2. Operating principle of a superconducting single photon detector. The white arrows denote the flow a supercurrent which is biasing the ultra-thin $(\sim 4 \mathrm{~nm}$ high by $\sim 100 \mathrm{~nm}$ wide) superconducting nanowire. A photon incident on the wire generates a resistive hot spot which suppresses the superconductivity at that region. The resulting voltage pulse can then be amplified.

The detection efficiency of NbN nanowire SSPDs may be increased by utilizing a cavity structure [9]. Recently a practical fiber coupling scheme for cavity embedded SSPDs has been demonstrated [10]. Optical coupling is via the thinned (45 $\mu \mathrm{m}$ thickness) $\mathrm{MgO}$ substrate and an Au mirror is placed atop the meander device. An NEP of $8.4 \times 10^{-18} \mathrm{~W} \mathrm{~Hz}^{-1 / 2}$ is reported at $1310 \mathrm{~nm}$ (practical detection efficiency $25 \%, 100 \mathrm{~Hz}$ dark count rate). An NEP of $1.9 \times 10^{-17} \mathrm{~W} \mathrm{~Hz}^{-1 / 2}$ is reported at $1550 \mathrm{~nm}$ (practical detection efficiency $9.5 \%, 100 \mathrm{~Hz}$ dark count rate).

To conclude, the field of single photon detection in the infrared regime has shown many advances in recent years. The stimulus of emerging photon-counting applications at the wavelength region around $1.55 \mu \mathrm{m}$ has driven the development of detectors capable of operating at these wavelengths. Research is concentrating on improving detection efficiency and reducing dark count rates, as well as improving packaging for practical system 
integration. It is likely that research will continue to progress in developing improved long wavelength detectors tailored to advanced infrared photon counting applications.

\section{References}

[1] G. S. Buller and R. J. Collins, "Single-photon generation and detection," J. Measure. Sci. Technol. 29, 012002 (2010) [doi:10.1088/0957-0233/21/1/012002].

[2] R. H. Hadfield, "Single photon detectors for optical quantum information applications," Nature Photon. 3, 696-705 (2009) [doi:10.1038/nphoton.2009.230].

[3] S. Pellegrini, R. E. Warburton, L. J. J. Tan, J. S. Ng, A. Krysa, K. Groom, J. P. R. David, S. D. Cova, M. J. Robertson, and G. S. Buller, "Design and performance of an InGaAs/InP single-photon avalanche diode detector," IEEE J. Quantum Electron. 42, 397-403 (2006) [doi:10.1109/JQE.2006.871067].

[4] Z. L. Yuan, B. E. Kardynal, A. W. Sharpe, and A. J. Shields, "High speed single photon detection in the near infrared," Appl. Phys. Lett. 91, 041114 (2007) [doi:10.1063/1.2760135].

[5] R. T. Thew, D. Stucki, J. D. Gautier, H. Zbinden, and A. Rochas, "Free-running InGaAs/InP avalanche photodiode with active quenching for single photon counting at telecom wavelengths," Appl. Phys. Lett. 91, 201114 (2007) [doi:10.1063/1.2815916].

[6] R.E. Warburton, M. Itzler, and G.S. Buller, "Improved free-running InGaAs/InP single-photon avalanche diode detectors operating at room temperature," Electronics Letters 45, 996-997 (2009) [doi:10.1049/el.2009.1508].

[7] G. N. Gol'tsman, O. Okunev, G. Chulkova, A. Lipatov, A. Semenov, K. Smirnov, B. Voronov, A. Dzardanov, C. Williams, and R. Sobolewski,. "Picosecond superconducting single-photon optical detector," Appl. Phys. Lett. 79, 705-707 (2001) [doi:10.1063/1.1388868].

[8] H. Takesue, S. W. Nam, Q. Zhang, R. H. Hadfield, T. Honjo, K. Tamaki and Y. Yamamoto, "Quantum key distribution over a 40-dB channel loss using superconducting single-photon detectors," Nature Photon. 1, 343 - 348 (2007) [doi:10.1038/nphoton.2007.75].

[9] K. M. Rosfjord, J. K. W. Yang, E. A. Dauler, A. Kerman, V. Anant, B. M. Voronov, G. N. Gol'tsman, and K K. Berggren, "Nanowire single-photon detector with an integrated optical cavity and anti-reflection coating," Opt. Exp. 14, 527-534 (2006) [doi:10.1364/OPEX.14.000527].

[10] S. Miki, M. Takeda, M. Fujiwara, M. Sasaski, and Z. Wang, "Compactly packaged superconducting nanowire single photon detector with an optical cavity for multichannel system" Opt. Exp.17, 23557- $23564 \quad$ (2009) [doi:10.1364/OE.17.023557].

Robert J. Collins is a research associate in the School of Engineering and Physical Sciences at Heriot-Watt University, UK. He graduated with an MPhys (Hons) degree in Physics in 2003 and, after a year spent in the commercial sector, a PhD in Physics in 2008. His areas of research include photon-counting and quantum information.

Robert H. Hadfield is a Reader in Physics and Royal Society University Research Fellow at Heriot-Watt University. He received his $\mathrm{PhD}$ degree from the University of Cambridge, UK in 2003 and spent four years as postdoctoral Guest Researcher at the US National Institute of Standards and Technology in Boulder, Colorado, USA. His research focuses on the development and applications of superconducting single photon detectors. 
Gerald S. Buller is a Professor of Physics at Heriot-Watt University, UK. He graduated with a BSc (Hons) degree in Natural Philosophy in 1986 and a PhD in Physics in 1989. He has co-authored more than 200 journal articles, conference papers, and patent applications in the areas of photon-counting detection and applications such as quantum key distribution and long-range depth imaging. He is also a director of Helia Photonics Limited, UK. 